DRAFT VERSION SEPTEMBER 28, 2021

Typeset using $\mathrm{LAT}_{\mathrm{E}} \mathrm{X}$ preprint style in AASTeX63

\title{
Energy and waiting time distributions of FRB 121102 observed by FAST
}

\author{
G. Q. Zhang, ${ }^{1}$ P. Wang, ${ }^{2}$ Q. Wu, ${ }^{1}$ F. Y. Wang,,${ }^{1,3}$ D. Li, ${ }^{2,4,5}$ Z. G. Dai, ${ }^{6,1}$ And B. Zhang ${ }^{7}$ \\ ${ }^{1}$ School of Astronomy and Space Science, Nanjing University, Nanjing 210093, China \\ ${ }^{2}$ CAS Key Laboratory of FAST, NAOC, Chinese Academy of Sciences, Beijing 100101, China \\ ${ }^{3}$ Key Laboratory of Modern Astronomy and Astrophysics (Nanjing University), Ministry of Education, Nanjing \\ 210093, China \\ ${ }^{4}$ University of Chinese Academy of Sciences, Beijing 100049, People's Republic of China \\ ${ }^{5}$ NAOC-UKZN Computational Astrophysics Centre, University of KwaZulu-Natal, Durban 4000, South Africa \\ ${ }^{6}$ Department of Astronomy, School of Physical Sciences, University of Science and Technology of China, Hefei \\ 230026, China \\ ${ }^{7}$ Department of Physics and Astronomy, University of Nevada, Las Vegas, Las Vegas, NV 89154, USA
}

Submitted to ApJL

\begin{abstract}
The energy and waiting time distributions are important properties for understanding the physical mechanism of repeating fast radio bursts (FRBs). Recently, the Fivehundred-meter Aperture Spherical radio Telescope (FAST, Nan et al. 2011; Li et al. 2018) detected the largest burst sample of FRB 121102, containing 1652 bursts Li et al. (2021a). We use this sample to investigate the energy and waiting time distributions. The energy count distribution $d N / d E$ at the high-energy range $\left(>10^{38} \mathrm{erg}\right)$ can be fitted with a single power-law function with an index of $-1.86_{-0.02}^{+0.02}$, while the distribution at the low-energy range deviates from the power-law function. An interesting result of Li et al. (2021a) is that there is an apparent temporal gap between early bursts (occurring before MJD 58740) and late bursts (occurring after MJD 58740). We find the energy distributions of high-energy bursts at different epochs are inconsistent. The power-law index is $-1.70_{-0.03}^{+0.03}$ for early bursts and $-2.60_{-0.14}^{+0.15}$ for late bursts. For bursts observed in a single day, a linear repetition pattern is found. We use the Weibull function to fit the distribution of waiting time of consecutive bursts. The shape parameter $k=0.72_{-0.01}^{+0.01}$ and the event rate $r=736.43_{-28.90}^{+26.55}$ day $^{-1}$ are derived. If the waiting times with $\delta_{t}<28 \mathrm{~s}$ are excluded, the burst behavior can be described by a Poisson process. The best-fitting values of $k$ are slightly different for low-energy $\left(E<1.58 \times 10^{38} \mathrm{erg}\right)$ and high-energy $\left(E>1.58 \times 10^{38} \mathrm{erg}\right)$ bursts.
\end{abstract}

Keywords: Radio bursts; Radio transient sources

Corresponding author: F. Y. Wang

fayinwang@nju.edu.cn 


\section{INTRODUCTION}

Fast radio bursts (FRBs) are bright bursts at radio frequency with milliseconds-duration (for reviews, see Cordes \& Chatterjee 2019; Petroff et al. 2019; Zhang 2020; Xiao et al. 2021). According to their burst activity, FRBs can be divided into two classes: repeating FRBs and apparently nonrepeating FRBs. There are more than twenty repeating FRBs detected so far, such as FRB 121102 (Spitler et al. 2016) and FRB 180916 (CHIME/FRB Collaboration et al. 2019). Whether all FRBs are repeating sources is still under debate (Palaniswamy et al. 2018; Caleb et al. 2019; Ravi 2019; Ai et al. 2021). The repeating activity excludes some cataclysmic models. Recently, FRB 200428 was found to be associated with a Galactic magnetar SGR 1935+2154 (CHIME/FRB Collaboration et al. 2020a; Bochenek et al. 2020), which supports that at least a part of FRBs is produced by magnetars. Some models have been proposed to interpret how magnetars may produce FRBs, such as magnetars in supernova remnants (Murase et al. 2016) or invoking shock interactions (Beloborodov 2017; Metzger et al. 2019), magnetars with low magnetospheric twist (Wadiasingh \& Timokhin 2019), and crust fracturing magnetars (Yang \& Zhang 2021).

FRB 121102 is the first detected repeating FRB, which has been observed from $400 \mathrm{MHz}$ to $8 \mathrm{GHz}$ (Spitler et al. 2014, 2016; Chatterjee et al. 2017; Michilli et al. 2018; Gajjar et al. 2018). In the past few years, about 300 bursts have been observed from this source. The high burst rate enables it to be investigated extensively. It was localized in a star-forming region in a dwarf galaxy in association with a persistent radio source (Chatterjee et al. 2017; Marcote et al. 2017; Tendulkar et al. 2017). The repeating bursts from FRB 121102 has an extreme high rotation measure (RM) of about $10^{5}$ $\mathrm{rad} \mathrm{m}^{-2}$ (Michilli et al. 2018), which is variable and decreasing (Michilli et al. 2018; Hilmarsson et al. 2021).

The energy distribution of repeating FRBs provides a clue to understanding the physical mechanism to produce FRBs. Wang \& Yu (2017) first found that the differential energy distribution of FRB 121102 is $d N / d E \propto E^{-1.8 \pm 0.15}$. Law et al. (2017) estimated that the energy distribution of FRB 121102 satisfies a single power-law distribution with $d N / d E \propto E^{-1.7}$. However, Gourdji et al. (2019) derived a power-law index of about $-2.8 \pm 0.3$. They argued that the differences between these results are caused by the incompleteness of the low energy. Wang \& Zhang (2019) used the cut-off powerlaw function to fit bursts observed by multiple observations and found that the power-law indices of different observations are close to -1.7 . The Apertif data suggested a slope of $-2.7 \pm 0.6$ above the completeness threshold $\sim 10^{39} \mathrm{erg}$ (Oostrum et al. 2020). An index $-2.1 \pm 0.1$ was found by Cruces et al. (2021) using the bursts detected by Effelsberg telescope. Therefore, the energy distribution of FRB 121102 has been controversial. To have a better understanding of the energy distribution, more bursts spanning a large energy range are required.

Although many bursts of FRB 121102 have been observed, the burst activity is still confusing. A possible long-term period has been discovered for FRB 121102. Rajwade et al. (2020) derived a period of about 156.9 days. This result was examined by Cruces et al. (2021). They found a period of about 161 days, which is consistent with the result of Rajwade et al. (2020). The similar periodic behavior (with a period of $\sim 16$ days) was discovered earlier for FRB 180916 (CHIME/FRB Collaboration et al. 2020b). Many models have been proposed to explain this periodic behavior, including binary star models (Dai et al. 2016; Ioka \& Zhang 2020; Dai \& Zhong 2020; Lyutikov et al. 2020; Deng et al. 2021; Kuerban et al. 2021; Wada et al. 2021; Li et al. 2021b), precession models 
(Zanazzi \& Lai 2020; Levin et al. 2020; Yang \& Zou 2020), and ultra-long period magnetar models (Beniamini et al. 2020).

No shorter period has been discovered for FRB 121102 (Zhang et al. 2018; Li et al. 2021a). Wang \& Yu (2017) first found that the repetition pattern of FRB 121102 cannot be described by a Poisson process. A similar conclusion was drawn by Oppermann et al. (2018). They also suggested that a Weibull function is suitable to describe the distribution of the waiting times of two consecutive bursts. The shape parameter $k$ is $0.34_{-0.05}^{+0.06}$, which means that the bursts of FRB 121102 tend to cluster in time. Oostrum et al. (2020) also used the Weibull function to fit the burst behavior and obtained $k=0.49 \pm 0.05$. A higher shape parameter $k=0.62_{-0.09}^{+0.10}$ was derived by Cruces et al. (2021). The values of $k$ are slightly different for multiple observations, which may be caused by different observing times, telescope sensitivities, or unknown periodic activities. A large sample with a long observing time and high sensitivity is required to clarify this issue.

Recently, the Five-hundred-meter Aperture Spherical radio Telescope (FAST) detected 1652 bursts from FRB 121102 ( $\mathrm{Li}$ et al. 2021a), which is the largest burst sample for this source. The high sensitivity of FAST ( Li et al. 2018) enables it to detect many low-energy bursts, which are difficult to detect with other telescopes. This large sample is helpful to reveal the properties of FRB 121102. Li et al. (2021a) reported bimodal energy distribution. More interestingly, there are three obvious peaks in the kernel density estimation (KDE) of energy and burst time (as shown in the panel (c) of their Figure 1). The high energy peak is only visible in early observations, while the low energy peaks exist in both early and late observations (Li et al. 2021a).

In this paper, we use FAST burst sample to investigate the energy distribution and burst activity of FRB 121102. This paper is organized as follows. In Section 2, we introduce the data and derive the energy distribution. The Weibull function is used to fit the waiting time distribution. The discussion is presented in Section 3 and conclusions are drawn in Section 4.

\section{DATA AND RESULTS}

\subsection{Data}

FRB 121102 has been observed by different telescopes (Spitler et al. 2014, 2016; Scholz et al. 2016, 2017; Chatterjee et al. 2017; Law et al. 2017; Hardy et al. 2017; Zhang et al. 2018; Spitler et al. 2018; Gajjar et al. 2018; Michilli et al. 2018; Gourdji et al. 2019; Hessels et al. 2019; Oostrum et al. 2020; Caleb et al. 2020; Cruces et al. 2021). Recently, FAST observed this source at $1.25 \mathrm{GHz}$ ( $\mathrm{Li}$ et al. 2021a). The observations were carried out from August 2019 (MJD 58724) to October 2019 (MJD 58776), a duration lasting nearly half of the $157 \mathrm{~d}$ period suggested by Rajwade et al. (2020) and covering almost the entire active phase (MJD 58717 to 58813) predicted by Cruces et al. (2021). During the 59.5 observing hours, FAST detected 1652 bursts. The mean burst rate was about 27.8 $\mathrm{hr}^{-1}$, and the peak burst rate was $122 \mathrm{hr}^{-1}$. These bursts help to understand the energy distribution in both the low-energy and high-energy ranges. The waiting time distribution can be studied in detail with this sample. We will use this sample to derive a more precise waiting time distribution.

\subsection{Energy Distribution}

The energy distribution of FRB 121102 has been investigated by many previous works (Wang \& Yu 2017; Law et al. 2017; Gourdji et al. 2019; Wang \& Zhang 2019; Cheng et al. 2020; Oostrum et al. 2020; Cruces et al. 2021; Li et al. 2021a). We use the bursts of FRB 121102 observed by FAST to 
investigate the differential energy count distribution

$$
d N / d E \propto E^{-\alpha_{E}} .
$$

The energies of these bursts are listed in Li et al. (2021a). They use central frequency rather than bandwidth to calculate the energies of bursts (This is to alleviate the bandwidth limitation of telescopes, see Zhang 2018). The luminosity distance of FRB 121102 is taken as 949 Mpc (Planck Collaboration \& et al. 2016; Tendulkar et al. 2017). We show the differential energy count distribution as a blue histogram in Figure 1. The distribution in the low energy range deviates from the power-law function. Therefore, to compare with previous works, we only consider the high-energy bursts $\left(E>10^{38} \mathrm{erg}\right)$, which contains roughly 600 bursts. The distribution can be approximated as a power-law function. We use equation (1) to fit the energy distribution in the high-energy end. The fitting result is shown as the green dot-dashed line in Figure 1. We also show the $90 \%$ threshold of FAST ( $\mathrm{Li}$ et al. 2021a) as vertical yellow dashed line in this figure. The power-law index is $\alpha_{E}=1.86 \pm 0.02$ ( $1 \sigma$ uncertainty), which is consistent with the index derived by Wang \& Yu (2017). Law et al. (2017) obtained a power-law index of $\alpha_{E} \simeq 1.7$, which is also consistent with our result. Wang \& Zhang (2019) used a cut-off power-law model to fit the FRB 121102 data from multiple observations and derived a universal energy distribution with $\alpha_{E}=1.6-1.8$ for multiple observations at varied frequencies. Our result also closes to this range. The energy distribution of high-energy bursts was also investigated by Li et al. (2021a). Because the observation of FAST for FRB 121102 consists of many single-day observations, Li et al. (2021a) used various observational times as the weight to derive the differential burst rate $d R / d E$ at different energies and found a power-law index of about $-1.85 \pm 0.3$. Our result is the distribution of burst counts in each energy bin $d N / d E$, not the burst rate distribution $d R / d E$.

Interestingly, the energies of non-repeating FRBs also show a power-law distribution with $\alpha_{E}=$ 1.6-1.8 (Cao et al. 2018; Lu \& Piro 2019; Zhang et al. 2019, 2021; Luo et al. 2018, 2020). Some sources also show a power-law distribution of $d N / d E$, such as X-ray bursts of magnetars (e.g. $\alpha_{E}=1.68 \pm 0.01$ for SGR 1806-20, Göğüs et al. 2000; Prieskorn \& Kaaret 2012; Cheng et al. 2020; Yang et al. 2021), X-ray flares of gamma-ray bursts $\left(\alpha_{E}=1.06 \pm 0.15\right.$, Wang \& Dai 2013), M87 $\left(\alpha_{E}=1.69_{-0.45}^{+0.59}\right.$, Yang et al. 2019) and Sgr A* $\left(\alpha_{E}=1.65 \pm 0.02\right.$, Wang et al. 2015; Li et al. 2015). Some FRB theoretical models are related to giant pulses of pulsars (e.g. Cordes \& Wasserman 2016). The giant pulses of Crab pulsar also show a power-law distribution $\left(\alpha_{E}=-1.85 \pm 0.10\right.$, Popov \& Stappers 2007; Lyu et al. 2021). A power-law distribution of energy is a natural predication of self-organized criticality theory (Bak et al. 1987; Aschwanden 2011).

The energy distribution in the low-energy range deviates from this power-law. This deviation was also reported by Gourdji et al. (2019). They attributed this deviation to the incompleteness at low energies. In our sample, the turning point is about $10^{38} \mathrm{erg}$, much larger than the threshold energy, which is about $3 \times 10^{37} \mathrm{erg}$ ( $\mathrm{Li}$ et al. 2021a). Therefore, this deviation is physical. A similar deviation was also found in other samples (Wang \& Zhang 2019; Oostrum et al. 2020; Cruces et al. 2021). Li et al. (2021a) also investigated the energy distribution of FRB 121102. They found the bimodal energy distribution, which is the Cauchy function with $\alpha_{E}=1.85 \pm 0.3$ for $E>10^{38}$ erg plus the $\log$-normal function with $\sigma_{E}=0.52, N=2.06 \times 10^{38}, E_{0}=7.2 \times 10^{37} \mathrm{erg}$ for $E<10^{38} \mathrm{erg}$. They also give the KDE of energy and burst time in the panel (c) of their Figure 1. In this panel, the high-energy component is only visible in early observations, while the low-energy peaks are present 
in both early and late observations. This novel structure may suggest different physical mechanisms for high-energy and low-energy bursts. If this conjecture is true, it can explain the deviation in low energies. The distribution also shows an obvious steepening above $E>5 \times 10^{39} \mathrm{erg}$, which indicates the burst maximum energy $E_{\max }$ is around this point.

\subsection{Waiting time Distribution}

Wang \& Yu (2017) found that the time interval between consecutive bursts does not follow a Poission distribution. Afterwards, Oppermann et al. (2018) found that the Weibull function is a better description of waiting time, which is described by

$$
\mathcal{W}\left(\delta_{t} \mid k, r\right)=k \delta_{t}^{-1}\left[\delta_{t} r \Gamma(1+1 / k)\right]^{k} \mathrm{e}^{-\left[\delta_{t} r \Gamma(1+1 / k)\right]^{k}},
$$

where $\delta_{t}$ is the waiting time, $k$ is the shape parameter, $r$ is the mean burst rate, and $\Gamma$ is the gamma function. The case $k=1$ corresponds to the Poisson distribution. Previous works suggested that the repeating behavior of FRB 121102 tends to have $k<1$ (Oppermann et al. 2018; Oostrum et al. 2020; Cruces et al. 2021), which means that time clustering is favored. We use the Weibull function to fit the burst behavior. The observations of FAST lasted for roughly 1 hour per day. Therefore, waiting time greater than a half day is caused by the observing window, which is ignored in our analysis. The waiting time with $\delta_{t}<30 \mathrm{~ms}$ is also excluded. It is difficult to determine whether they are two separate bursts or multiple peaks of a single burst (Cruces et al. 2021). We use the MCMC (Markov chain Monte Carlo) technique to fit this distribution. The corner plot of the best-fitting results is shown in Figure 2. We list the best-fitting parameters in Table 1, alongside the results from previous works. We find $k=0.72_{-0.01}^{+0.01}\left(1 \sigma\right.$ uncertainty) and $r=736.43_{-28.90}^{+26.55}$ day $^{-1}$ ( $1 \sigma$ uncertainty). This burst rate is much higher than the rates derived in previous works, which is caused by the high sensitivity of FAST. The $90 \%$ threshold of FAST sample is $2.5 \times 10^{37}$ erg (Li et al. 2021a).. The cumulative distribution of waiting time is shown in Figure 3. The green dashed line is the predicted result of the Weibull function, the dot-dashed red line is predicted by a Poisson process, and the black dotted line is the best-fitting results of the log-normal distribution. Some previous works suggest log-normal distribution is suitable to fit the waiting time distribution (Gourdji et al. 2019; Katz 2019). Li et al. (2021a) used two log-normal functions to fit the waiting time distribution. We add the fit of log-normal distribution as a reference and do not discuss it in detail. The $\chi^{2}$ values for Poisson distribution, Weibull distribution, and log-normal distribution are 392.28, 346.50, and 394.90, respectively. The Weibull function is the best in fitting the data. In Figure 3, Poisson and log-normal distributions cannot well describe short waiting times. However, the Weibull distribution has a better description of that. For long waiting times, both the Weibull distribution and log-normal distribution can model the data well.

Oppermann et al. (2018) collected 17 bursts observed by Arecibo, Effelsberg, GBT, VLA, and Lovell. They derived $k=0.34_{-0.05}^{+0.06}$ with these 17 bursts. Oostrum et al. (2020) obtained $k=$ $0.49 \pm 0.05$. Their results are smaller than ours. This may be caused by the low waiting time cutoff. Cruces et al. (2021) found that when excluding the waiting time $\delta_{t}<1 \mathrm{~s}$, the shape parameter $k$ shifts from $k=0.62_{-0.09}^{+0.10}$ to $k=0.73_{-0.10}^{+0.12}$. We carefully check the effect of waiting time cutoff $\delta_{t, c}$ and show the dependence of $k$ on $\delta_{t, c}$ in Figure 4. The solid blue line is the evolution of $k$ and the vertical solid blue lines are $1 \sigma$ errors. The shape parameter $k$ increases as $\delta_{t, c}$ increases. When $\delta_{t, c} \simeq 28 s$, the shape parameter $k$ closes to 1 , which means that the repeating behavior is due to a Poisson process. 
Cruces et al. (2021) also found that the waiting time with $\delta_{t}>100 \mathrm{~s}$ is consistent with a Poisson process, which is similar to our results.

\subsection{Linear repetition pattern}

We use the FAST data to check the burst repetition pattern. We select the single-day observations with the number of detected bursts larger than 60 and show the cumulative distribution function of the burst time with blue scatters in each panel of Figure 5. The observation time is listed in the top of each panel. The x-axis is the decimal part of the MJD time. We find that the distribution can be fitted with a linear function

$$
N(<t)=s t+b,
$$

where $t$ is the burst time, $s$ is the slope, and $b$ is a constant. The best-fitting results with the dashed red lines are shown in each panel and the best-fitting parameters are also listed. The observation on 2019-09-07 is slightly different from other observations. There is an apparent temporal gap between early bursts occurring before MJD 58732.90 and late ones occurring after MJD 58732.90. In this observation, most bursts occurred after MJD 58732.90 and these bursts can be fitted with Equation (3). For all the observations listed in Figure 5, although the fitting results are different, they can be all fitted with equation 3. Tabor \& Loeb (2020) proposed that the burst rate is constant per logarithmic time with the observation of GBT (Zhang et al. 2018). They found that the cumulative distribution function can be fitted with $N(<t)=\alpha \ln \left(t / t_{0}\right)$. Contrary, our results suggest that FAST strongly favors a linear pattern. Tabor \& Loeb (2020) excluded the model that repeating FRB sources origin from pulsars (Beniamini et al. 2020). According to our results, this model is still feasible. The linear pattern may suggest a potentially short period. However, similar to Li et al. (2021a), we do not find any short period. The Lomb-Scargle method and epoch folding method are used to search between 10 milliseconds and 30 minutes. No significant period was found. The fluctuations of data points along the fitted lines in Figure 5 may suggest that the linear pattern is caused by the narrow waiting time distribution. Besides, the slopes in varied observations are different, which may suggest diverse waiting time distribution in different observations.

\section{DISCUSSION}

\subsection{Energy distribution in different epochs}

An important conclusion of Li et al. (2021a) is the novel triple peaks structure in the KDE of energy and burst time (As shown in the panel (c) of their Figure 1). There is a clear energy gap between the high-energy bursts and low-energy bursts before MJD 58740. The temporal gap between early bursts and late bursts is also significant. Following the division of Li et al. (2021a), we regard the bursts occurring before MJD 58740 as early bursts and the bursts occurring after MJD 58740 as late bursts. The energy distributions of early bursts and late bursts are shown in Figure 6. Again, we only fit the bursts with energy $>10^{38} \mathrm{erg}$. The best-fitting results are shown as green dot-dashed

lines. The power-law index for the early bursts is $-1.70_{-0.03}^{+0.03}$ ( $1 \sigma$ uncertainty), while that for the late bursts is $-2.60_{-0.14}^{+0.15}$ ( $1 \sigma$ uncertainty), which is significantly different.

Some previous works support the power-law index -1.7 (Wang \& Yu 2017; Law et al. 2017; Wang \& Zhang 2019), which is consistent with the result of early bursts. However, there are also some works suggest a steeper index (Gourdji et al. 2019), which is close to the result of late bursts. This difference may be caused by the following reasons. There may be different modes of burst emission 
operating in different epochs, so that the observed energy distribution may depend on the observing time. The two different modes may be related to different mechanisms or different emission sites. Li et al. (2021a) reported the triple peaks in the distribution of energy and burst time (as shown in panel (c) of their Figure 1). At the early epoch, the bursts with $E>10^{38}$ erg belong to the high-energy burst component. However, the high-energy peak is invisible in the late epoch. The bursts with $E>10^{38} \mathrm{erg}$ in the late epoch are an extension of the low-energy peak. If the high-energy bursts and low-energy bursts originate from different mechanisms or different sites, which operate in different epochs, the variance of the energy distribution is naturally interpreted.

\subsection{Waiting time for different energies}

Li et al. (2021a) reported the triple peak structure in the panel (c) of their Figure 1. The bimodal structure in the energy distribution in the early phase (before MJD 58740) may suggest that they are produced by different physical processes. Therefore, we explore whether there are differences in their other properties, such as waiting time. If the low-energy bursts and high-energy bursts are produced by different processes, the waiting time should be calculated independently. We divide all bursts into three sub-samples: the low-energy bursts and high-energy bursts between MJD 58717 and 58740, and the late-phase bursts detected between MJD 58740 and 58776. The dividing line of burst time is consistent with the choice of Li et al. (2021a). We take the dividing line between the low-energy bursts and high-energy bursts as $1.58 \times 10^{38} \mathrm{erg}$. The gap is about $10^{38.1} \sim 10^{38.3} \mathrm{erg}$. For convenience, we take the separation line as $10^{38.2} \simeq 1.58 \times 10^{38} \mathrm{erg}$. We also test other choices of the criteria and find that it does not affect on our result. For each sub-sample we calculate the waiting time independently and show the KDE of the waiting time in Figure 7. The KDE for these three sub-samples is slightly different. The medians of the low-energy bursts, high-energy bursts, and late-bursts are $78.19 \mathrm{~s}, 133.61 \mathrm{~s}$, and $54.62 \mathrm{~s}$, respectively. There is a small peak in the KDE density of the late-phase bursts, which is close to $0.1 \mathrm{~s}$.

We also use the Weibull function to fit three sub-samples. Again, we ignore the waiting times with $\delta_{t}<30 \mathrm{~ms}$ and $\delta_{t}>0.5$ day. We list the best-fitting results for three sub-samples in Table 1 . The shape parameters are $k \simeq 0.69 \pm 0.02$ for the low-energy bursts, $k \simeq 0.76 \pm 0.03$ for the high-energy bursts and $k \simeq 0.66 \pm 0.02$ for the late-phase bursts. The posterior distributions of $k$ for the three sub-samples are shown in Figure 8. The $k$ parameters for the low-energy bursts, high-energy bursts, and late-phase bursts are shown as black line, red line, and blue line, respectively. The vertical dashed lines are the best-fitting results. The shape parameters $k$ for the low-energy and late-phase bursts are consistent with each other within $1 \sigma$ error, while the $k$ for the high-energy bursts is higher. The consistency of the low-energy bursts and late-phase bursts may suggest that they have the same origin, while the high-energy bursts may have a different origin. The difference between the latephase bursts and high-energy bursts is significant, but the variance between the low-energy bursts and high-energy bursts is insignificant. This may be caused by the rough $1.58 \times 10^{38} \mathrm{erg}$ division line of low-energy bursts and high-energy bursts. The count distributions of low-energy and high-energy bursts are close to a log-normal distribution. They can be extended to include each other. Therefore, some FRBs may be misclassified. If we can make a robust classification, the difference in waiting time may be larger.

We derive the burst rates for the low-energy bursts and high-energy bursts are $445.35_{-30.02}^{+31.85}$ per day and $354.74_{-26.14}^{+27.14}$ per day, respectively. The burst rate of the low-energy bursts is higher than that of the high energy bursts. These two classes of bursts occurred in the same epoch. For the bursts 
occurring in late observation days, the burst rate is about $800.54_{-48.32}^{+48.84}$ per day, which is higher than that of low-energy bursts and high-energy bursts, but close to the sum of these two classes of bursts.

We also check the dependence of energy on waiting time. For X-ray flares of gamma-ray bursts, an anti-correlation is found between energy and waiting time (Yi et al. 2016). The scatter plot of energy and waiting time of the FAST FRB sample is shown in Figure 9. No significant dependence of energy on waiting time is found. We also check the dependence for three sub-samples and found no significant dependence.

\subsection{Waiting time in different observational days}

The FAST observations spanned two months in time, which covered most of the active window of FRB 121102. We can test the dependence of the burst behaviors on observing time. The observations lasted for roughly 1 hour each day. We regard the observations in each day as single samples and select some samples with the number of observed bursts greater than 5 . Then, the Weibull function is applied to fit the waiting time distribution in these days. The best-fitting results against burst time are shown in Figure 10. The blue points are the shape parameters $k$ with $1 \sigma$ errors, and the yellow points are the burst rates $r$ with $1 \sigma$ errors. Interestingly, the value of $k$ is close to 1 in some days, which indicates a rough Poisson distribution of the waiting time. Some observations have $k>1$, which means that the waiting time prefers a specific value. If $k \gg 1$, it means a periodic behavior. In our calculation, the value of $k$ is not enough to support the periodic behavior. It only suggests that the waiting time has a narrow distribution in some days, which is consistent with the linear pattern. The best-fitting result varies for different samples. We do not find significant dependence on the observation time.

\section{CONCLUSIONS}

The energy and waiting time distributions of FRB 121102 provide a clue for understanding the physical origin of repeating FRBs. Li et al. (2021a) reported a large sample of bursts (1652), about 1200 of which are faint bursts with $E<1.58 \times 10^{38} \mathrm{erg}$ (the nominal threshold for separating low and high energy events). The detection of these faint bursts enables us to perform extensive statistical studies of the burst properties. Our conclusions can be summarized as follows.

1. The energy distribution in the high-energy range can be fitted with a simple power-law function. The power-law index is $-1.86 \pm 0.02$, which is consistent with previous results (Wang \& Yu 2017; Law et al. 2017; Wang \& Zhang 2019; Cruces et al. 2021). This index is also similar to those of non-repeating FRBs (Cao et al. 2018; Lu \& Piro 2019) and magnetar X-ray bursts (Göğüş et al. 2000; Cheng et al. 2020), which may suggest they share similar physical processes. However, the energy distribution deviates from this power-law function at low energies. The turning point is near $10^{38} \mathrm{erg}$, well above the $90 \%$ detection completeness threshold $\left(E_{90}=2.5 \times 10^{37} \mathrm{erg}\right)$. Thus, this deviation is not caused by the incompleteness in the low-energy end. A different physical mechanism or different emission site for low-energy events may be required to explain this deviation.

2. The Weibull function is used to fit the waiting times excluding events separated by $\delta_{t}<30 \mathrm{~ms}$ and $\delta_{t}>0.5$ day. We derive $k=0.72_{-0.01}^{+0.01}$ and $r=736.43_{-28.90}^{+26.55}$ day $^{-1}$. This result $(k<1)$ suggests that the bursts of FRB 121102 tend to cluster in time. We also found that when we 
exclude the waiting time $\delta_{t}<28 \mathrm{~s}$, the burst behavior can be roughly described by a Poisson process.

3. For bursts observed in a single day, a linear repetition pattern of burst time is found. We select the observational days when more than 60 bursts were detected. The cumulative distributions of the burst time are shown in Figure 5. These distributions can be fitted with $N(<t)=s t+b$.

4. The distributions of high-energy early bursts and late bursts are different.The dividing line of early bursts and late bursts is MJD 58740. When the bursts with $E>10^{38} \mathrm{erg}$ are considered, the power-law index is -1.70 for early bursts and -2.60 for late bursts. This difference may suggest that they have different physical origins.

5. According to the bimodal structure of energy distribution reported by Li et al. (2021a), we divide the bursts into 3 classes: the low-energy bursts and high-energy bursts between MJD 58717 and 58740, and the late-phase bursts. The distributions of waiting time for these three classes are somewhat different. We also use the Weibull function to fit the waiting time distributions. The posterior probability distribution of the $k$ parameter for the high-energy bursts is slightly different from that of the low-energy and late-phase bursts. These differences may suggest that they have different physical origins or emission sites.

6. There is no significant dependence of energy on waiting time. For three sub-samples, we also do not find any dependence between the two parameters.

\section{ACKNOWLEDGEMENTS}

We would like to thank the anonymous referee for helpful comments. This work was supported by the National Natural Science Foundation of China (grant No. 11988101, No. U1831207, No. 11833003, No. 11725313, No. 11690024, No. 12041303 and No. U2031117), the Fundamental Research Funds for the Central Universities (No. 0201-14380045), the National Key Research and Development Program of China (grant 2017YFA0402600); and the National SKA Program of China No. 2020SKA0120200, the Cultivation Project for FAST Scientific Payoff and Research Achievement of CAMS-CAS. PW acknowledges support by the Youth Innovation Promotion Association CAS (id. 2021055) and CAS Project for Young Scientists in Basic Reasearch (grant YSBR-006). This work made use of data from FAST, a Chinese national mega-science facility built and operated by the National Astronomical Observatories, Chinese Academy of Sciences.

\section{REFERENCES}

Ai, S., Gao, H., \& Zhang, B. 2021, ApJL, 906, L5, doi: $10.3847 / 2041-8213 /$ abcec9

Aschwanden, M. J. 2011, Self-Organized Criticality in Astrophysics

Bak, P., Tang, C., \& Wiesenfeld, K. 1987, PhRvL, 59, 381, doi: 10.1103/PhysRevLett.59.381

Beloborodov, A. M. 2017, ApJL, 843, L26, doi: $10.3847 / 2041-8213 /$ aa78f3
Beniamini, P., Wadiasingh, Z., \& Metzger, B. D. 2020, MNRAS, 496, 3390, doi: 10.1093/mnras/staa1783

Bochenek, C. D., Ravi, V., Belov, K. V., et al. 2020, Nature, 587, 59, doi: 10.1038/s41586-020-2872-x

Caleb, M., Stappers, B. W., Rajwade, K., \& Flynn, C. 2019, MNRAS, 484, 5500, doi: $10.1093 / \mathrm{mnras} / \mathrm{stz} 386$ 
Caleb, M., Stappers, B. W., Abbott, T. D., et al. 2020, MNRAS, 496, 4565, doi: $10.1093 / \mathrm{mnras} / \mathrm{staa} 1791$

Cao, X.-F., Yu, Y.-W., \& Zhou, X. 2018, ApJ, 858, 89, doi: 10.3847/1538-4357/aabadd

Chatterjee, S., Law, C. J., Wharton, R. S., et al. 2017, Nature, 541, 58, doi: 10.1038/nature20797

Cheng, Y., Zhang, G. Q., \& Wang, F. Y. 2020, MNRAS, 491, 1498, doi: 10.1093/mnras/stz3085

CHIME/FRB Collaboration, Andersen, B. C., Bandura, K., et al. 2019, ApJL, 885, L24, doi: 10.3847/2041-8213/ab4a80

CHIME/FRB Collaboration, Andersen, B. C., Bandura, K. M., et al. 2020a, Nature, 587, 54, doi: 10.1038/s41586-020-2863-y

CHIME/FRB Collaboration, Amiri, M., Andersen, B. C., et al. 2020b, Nature, 582, 351, doi: $10.1038 / \mathrm{s} 41586-020-2398-2$

Cordes, J. M., \& Chatterjee, S. 2019, ARA\&A, 57, 417, doi: 10.1146/annurev-astro-091918-104501

Cordes, J. M., \& Wasserman, I. 2016, MNRAS, 457, 232, doi: 10.1093/mnras/stv2948

Cruces, M., Spitler, L. G., Scholz, P., et al. 2021, MNRAS, 500, 448, doi: 10.1093/mnras/staa3223

Dai, Z. G., Wang, J. S., Wu, X. F., \& Huang, Y. F. 2016, ApJ, 829, 27, doi: 10.3847/0004-637X/829/1/27

Dai, Z. G., \& Zhong, S. Q. 2020, ApJL, 895, L1, doi: $10.3847 / 2041-8213 / a b 8 f 2 d$

Deng, C.-M., Zhong, S.-Q., \& Dai, Z.-G. 2021, arXiv e-prints, arXiv:2102.06796. https://arxiv.org/abs/2102.06796

Gajjar, V., Siemion, A. P. V., Price, D. C., et al. 2018, ApJ, 863, 2, doi: $10.3847 / 1538-4357 /$ aad005

Gourdji, K., Michilli, D., Spitler, L. G., et al. 2019, ApJL, 877, L19, doi: 10.3847/2041-8213/ab1f8a

Göğüss, E., Woods, P. M., Kouveliotou, C., et al. 2000, ApJL, 532, L121, doi: 10.1086/312583

Hardy, L. K., Dhillon, V. S., Spitler, L. G., et al. 2017, MNRAS, 472, 2800, doi: $10.1093 /$ mnras/stx2153

Hessels, J. W. T., Spitler, L. G., Seymour, A. D., et al. 2019, ApJL, 876, L23, doi: 10.3847/2041-8213/ab13ae

Hilmarsson, G. H., Michilli, D., Spitler, L. G., et al. 2021, ApJL, 908, L10, doi: $10.3847 / 2041-8213 /$ abdec0

Ioka, K., \& Zhang, B. 2020, ApJL, 893, L26, doi: $10.3847 / 2041-8213 / a b 83 f b$
Katz, J. I. 2019, MNRAS, 487, 491, doi: $10.1093 / \mathrm{mnras} / \mathrm{stz} 1250$

Kuerban, A., Huang, Y.-F., Geng, J.-J., et al. 2021, arXiv e-prints, arXiv:2102.04264. https://arxiv.org/abs/2102.04264

Law, C. J., Abruzzo, M. W., Bassa, C. G., et al. 2017, ApJ, 850, 76, doi: $10.3847 / 1538-4357 /$ aa9700

Levin, Y., Beloborodov, A. M., \& Bransgrove, A. 2020, ApJL, 895, L30, doi: 10.3847/2041-8213/ab8c4c

Li, D., Wang, P., Qian, L., et al. 2018, IEEE Microwave Magazine, 19, 112, doi: 10.1109/MMM.2018.2802178

Li, D., Wang, P., Zhu, W. W., et al. 2021a, arXiv e-prints, arXiv:2107.08205. https://arxiv.org/abs/2107.08205

Li, Q.-C., Yang, Y.-P., Wang, F. Y., et al. 2021b, arXiv e-prints, arXiv:2108.00350. https://arxiv.org/abs/2108.00350

Li, Y.-P., Yuan, F., Yuan, Q., et al. 2015, ApJ, 810, 19, doi: 10.1088/0004-637X/810/1/19

Lu, W., \& Piro, A. L. 2019, ApJ, 883, 40, doi: $10.3847 / 1538-4357 / a b 3796$

Luo, R., Lee, K., Lorimer, D. R., \& Zhang, B. 2018, MNRAS, 481, 2320, doi: 10.1093/mnras/sty2364

Luo, R., Men, Y., Lee, K., et al. 2020, MNRAS, 494, 665, doi: 10.1093/mnras/staa704

Lyu, F., Meng, Y.-Z., Tang, Z.-F., et al. 2021, Frontiers of Physics, 16, 24503, doi: 10.1007/s11467-020-1039-4

Lyutikov, M., Barkov, M. V., \& Giannios, D. 2020, ApJL, 893, L39, doi: 10.3847/2041-8213/ab87a4

Marcote, B., Paragi, Z., Hessels, J. W. T., et al. 2017, ApJL, 834, L8, doi: 10.3847/2041-8213/834/2/L8

Metzger, B. D., Margalit, B., \& Sironi, L. 2019, MNRAS, 485, 4091, doi: 10.1093/mnras/stz700

Michilli, D., Seymour, A., Hessels, J. W. T., et al. 2018, Nature, 553, 182, doi: 10.1038/nature25149

Murase, K., Kashiyama, K., \& Mészáros, P. 2016, MNRAS, 461, 1498, doi: $10.1093 /$ mnras $/$ stw 1328

Nan, R., Li, D., Jin, C., et al. 2011, International Journal of Modern Physics D, 20, 989, doi: 10.1142/S0218271811019335 
Oostrum, L. C., Maan, Y., van Leeuwen, J., et al. 2020, A\&A, 635, A61, doi: 10.1051/0004-6361/201937422

Oppermann, N., Yu, H.-R., \& Pen, U.-L. 2018, MNRAS, 475, 5109, doi: 10.1093/mnras/sty004

Palaniswamy, D., Li, Y., \& Zhang, B. 2018, ApJL, 854, L12, doi: 10.3847/2041-8213/aaaa63

Petroff, E., Hessels, J. W. T., \& Lorimer, D. R. 2019, A\&A Rv, 27, 4, doi: 10.1007/s00159-019-0116-6

Planck Collaboration, \& et al. 2016, A\&A, 594, A13, doi: 10.1051/0004-6361/201525830

Popov, M. V., \& Stappers, B. 2007, A\&A, 470, 1003, doi: 10.1051/0004-6361:20066589

Prieskorn, Z., \& Kaaret, P. 2012, ApJ, 755, 1, doi: 10.1088/0004-637X/755/1/1

Rajwade, K. M., Mickaliger, M. B., Stappers, B. W., et al. 2020, MNRAS, 495, 3551, doi: $10.1093 / \mathrm{mnras} / \mathrm{staa} 1237$

Ravi, V. 2019, Nature Astronomy, 405, doi: 10.1038/s41550-019-0831-y

Scholz, P., Spitler, L. G., Hessels, J. W. T., et al. 2016, ApJ, 833, 177, doi: $10.3847 / 1538-4357 / 833 / 2 / 177$

Scholz, P., Bogdanov, S., Hessels, J. W. T., et al. 2017, ApJ, 846, 80, doi: $10.3847 / 1538-4357 /$ aa 8456

Spitler, L. G., Cordes, J. M., Hessels, J. W. T., et al. 2014, ApJ, 790, 101, doi: 10.1088/0004-637X/790/2/101

Spitler, L. G., Scholz, P., Hessels, J. W. T., et al. 2016, Nature, 531, 202, doi: $10.1038 /$ nature17168

Spitler, L. G., Herrmann, W., Bower, G. C., et al. 2018, ApJ, 863, 150, doi: $10.3847 / 1538-4357 / \operatorname{aad} 332$

Tabor, E., \& Loeb, A. 2020, ApJL, 902, L17, doi: 10.3847/2041-8213/abba79

Tendulkar, S. P., Bassa, C. G., Cordes, J. M., et al. 2017, ApJL, 834, L7, doi: $10.3847 / 2041-8213 / 834 / 2 /$ L7
Wada, T., Ioka, K., \& Zhang, B. 2021, arXiv e-prints, arXiv:2105.14480. https://arxiv.org/abs/2105.14480

Wadiasingh, Z., \& Timokhin, A. 2019, ApJ, 879, 4, doi: $10.3847 / 1538-4357 /$ ab2240

Wang, F. Y., \& Dai, Z. G. 2013, Nature Physics, 9, 465, doi: 10.1038/nphys2670

Wang, F. Y., Dai, Z. G., Yi, S. X., \& Xi, S. Q. 2015, ApJS, 216, 8, doi: 10.1088/0067-0049/216/1/8

Wang, F. Y., \& Yu, H. 2017, JCAP, 03, 023, doi: 10.1088/1475-7516/2017/03/023

Wang, F. Y., \& Zhang, G. Q. 2019, ApJ, 882, 108, doi: $10.3847 / 1538-4357 / a b 35 d c$

Xiao, D., Wang, F., \& Dai, Z. 2021, arXiv e-prints, arXiv:2101.04907. https://arxiv.org/abs/2101.04907

Yang, H., \& Zou, Y.-C. 2020, ApJL, 893, L31, doi: 10.3847/2041-8213/ab800f

Yang, S., Yan, D., Dai, B., et al. 2019, MNRAS, 489, 2685, doi: 10.1093/mnras/stz2302

Yang, Y.-H., Zhang, B.-B., Lin, L., et al. 2021, ApJL, 906, L12, doi: 10.3847/2041-8213/abd02a

Yang, Y.-P., \& Zhang, B. 2021, arXiv e-prints, arXiv:2104.01925. https://arxiv.org/abs/2104.01925

Yi, S.-X., Xi, S.-Q., Yu, H., et al. 2016, ApJS, 224, 20, doi: 10.3847/0067-0049/224/2/20

Zanazzi, J. J., \& Lai, D. 2020, ApJL, 892, L15, doi: $10.3847 / 2041-8213 /$ ab7cdd

Zhang, B. 2018, ApJL, 867, L21, doi: $10.3847 / 2041-8213 /$ aae8e3

—. 2020, Nature, 587, 45, doi: 10.1038/s41586-020-2828-1

Zhang, G. Q., Wang, F. Y., \& Dai, Z. G. 2019, arXiv e-prints, arXiv:1903.11895. https://arxiv.org/abs/1903.11895

Zhang, R. C., Zhang, B., Li, Y., \& Lorimer, D. R. 2021, MNRAS, 501, 157, doi: 10.1093/mnras/staa3537

Zhang, Y. G., Gajjar, V., Foster, G., et al. 2018, ApJ, 866, 149, doi: 10.3847/1538-4357/aadf31 


\begin{tabular}{|l|l|l|}
\hline Data set & $r\left(\right.$ day $\left.^{-1}\right)$ & $k$ \\
\hline All bursts of Li et al. (2021a) & $736.43_{-28.90}^{+26.55}$ & $0.72_{-0.01}^{+0.01}$ \\
\hline low-energy bursts & $445.35_{-31.59}^{+30.42}$ & $0.69_{-0.02}^{+0.02}$ \\
\hline high-energy bursts & $354.74_{-26.14}^{+27.14}$ & $0.76_{-0.03}^{+0.03}$ \\
\hline late-phase bursts & $800.54_{-48.32}^{+48.84}$ & $0.66_{-0.02}^{+0.02}$ \\
\hline Oppermann et al. (2018) & $5.7_{-2.0}^{+3.0}$ & $0.34_{-0.05}^{+0.06}$ \\
\hline Oostrum et al. (2020) & $6.9_{-1.5}^{+1.9}$ & $0.49_{-0.05}^{+0.05}$ \\
\hline Cruces et al. (2021) & $74_{-22}^{+31}$ & $0.62_{-0.09}^{+0.10}$ \\
\hline
\end{tabular}

Table 1. The best-fitting results of Weibull distribution for different samples. The classification of the lowenergy bursts, high-energy bursts, and late-phase bursts is given in Section 3.2. We exclude waiting times with $\delta_{t}>0.5$ day and $\delta_{t}<30 \mathrm{~ms}$ from all bursts, low-energy bursts, high-energy bursts, and late-phase bursts. The results from other works are listed for reference.

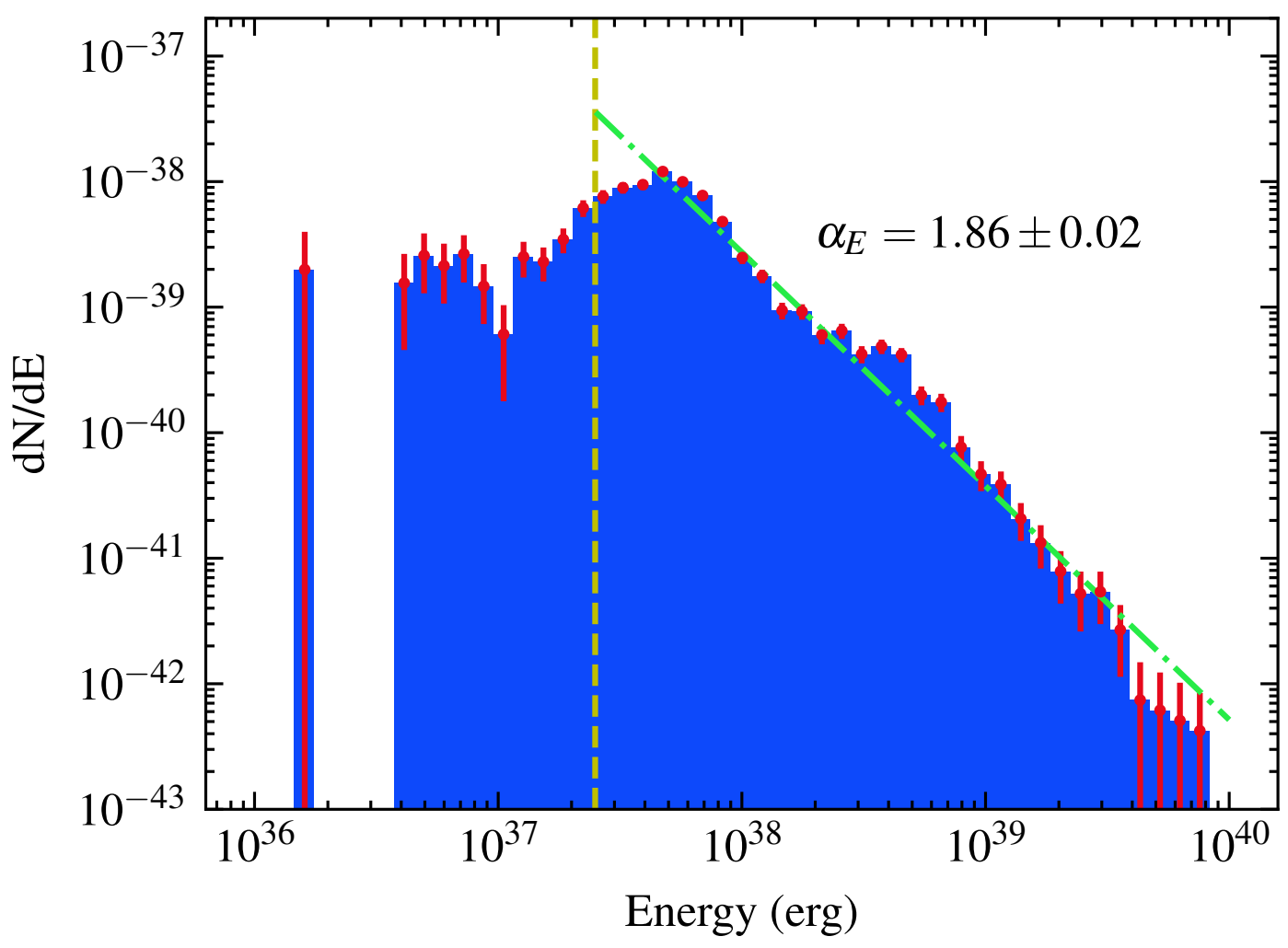

Figure 1. The differential energy distribution $d N / d E$ for the bursts of FRB 121102 observed by Li et al. (2021a). The blue histogram is the differential energy distribution and the red points and red vertical lines are the values and $1 \sigma$ uncertainties. The vertical yellow dashed line is the $90 \%$ completeness threshold of FAST telescope ( $\mathrm{Li}$ et al. 2021a). We use the simple power-law (equation 1) to fit the energy distribution in the high-energy range $\left(E>10^{38} \mathrm{erg}\right)$ and show the best-fitting result as dot-dashed green line. The power-law index is $-1.86 \pm 0.02$. The bursts in the low energies deviate from power-law form. 


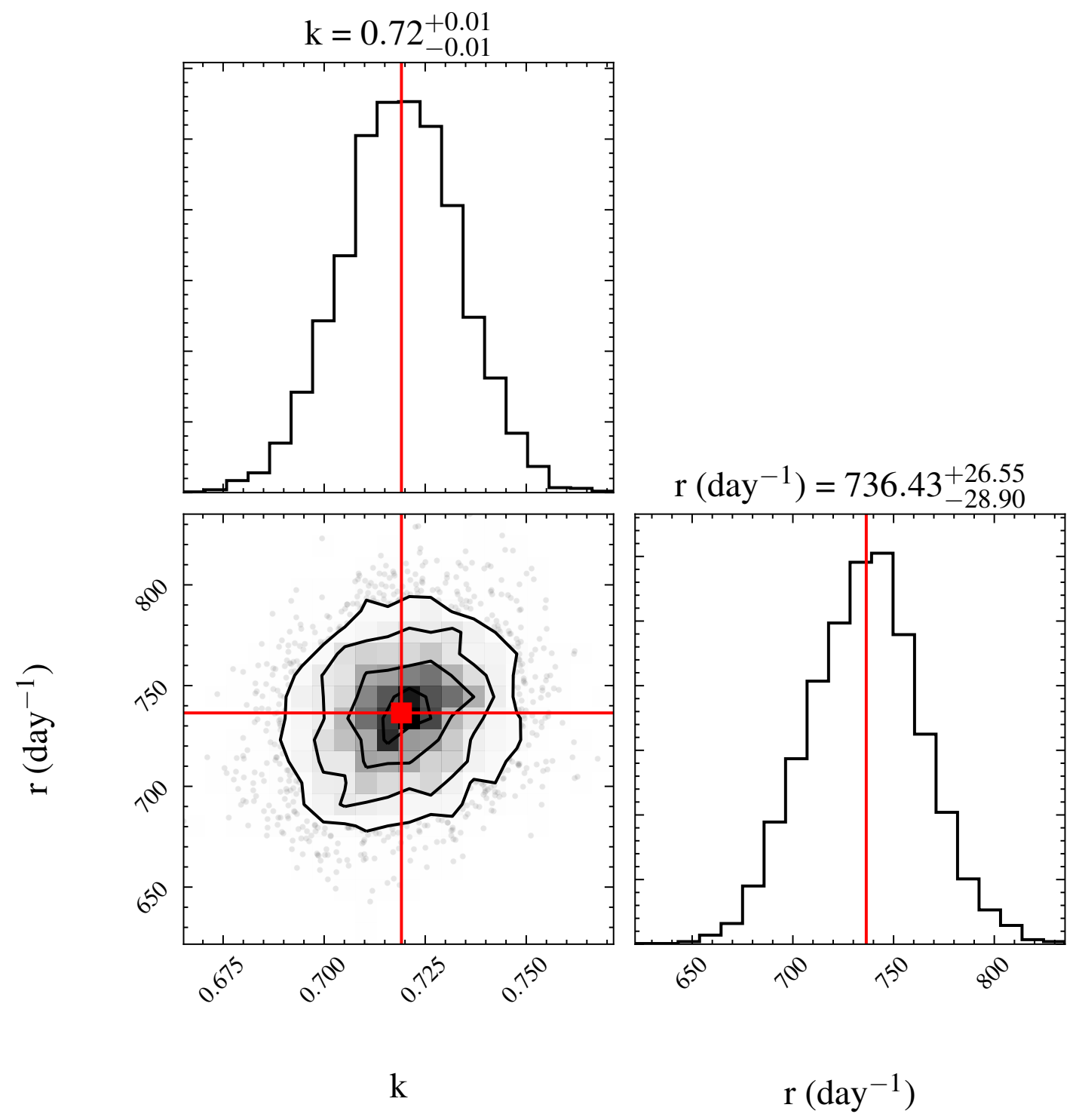

Figure 2. The corner plot for the shape parameter $k$ and event rate $r$ for the Weibull distribution. The waiting times with $\delta_{t}<30 \mathrm{~ms}$ and $\delta_{t}>0.5$ day are excluded. We use the red lines to denote the best-fitting results, which are $k=0.72_{-0.01}^{+0.01}$ and $r=736.43_{-28.90}^{+26.55}$ per day. 


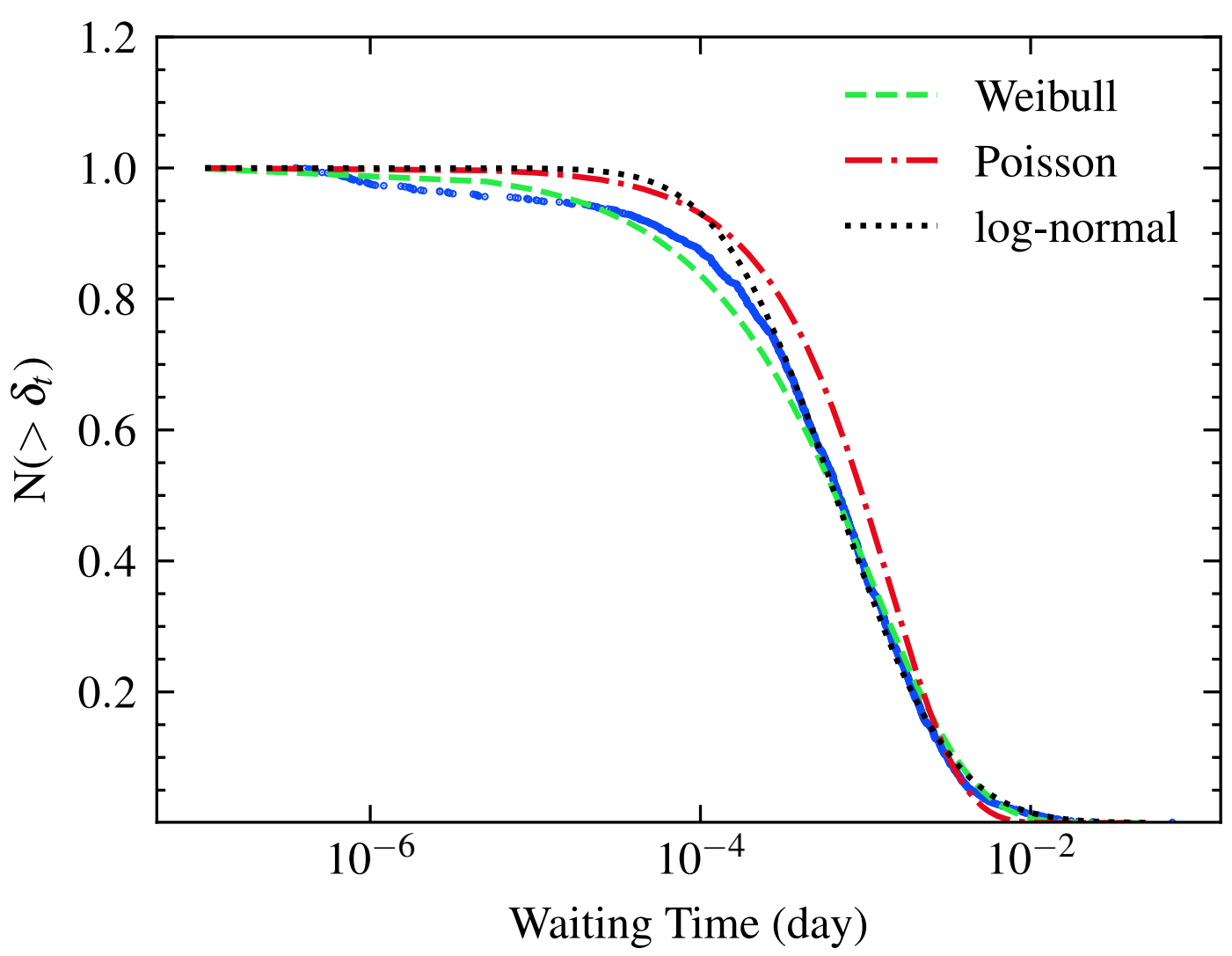

Figure 3. The cumulative distribution of the waiting time between consecutive bursts. The blue scatter is the burst waiting time distribution. The dashed green line, dot-dashed red line, and dotted black line are best-fitting results of Weibull distribution, Poisson distribution, and log-normal distribution, respectively. The best-fitting results are $k=0.72_{-0.01}^{+0.01}$ and $r=736.43_{-28.90}^{+26.55}$ per day for Weibull distribution, $r=722.90$ per day for Poisson distribution, and $\mu=-7.33, \sigma=1.26$ for log-normal distribution. The $\chi^{2}$ for Weibull function, Poisson function and log-normal function are 346.50,392.28 and 394.90, respectively. 


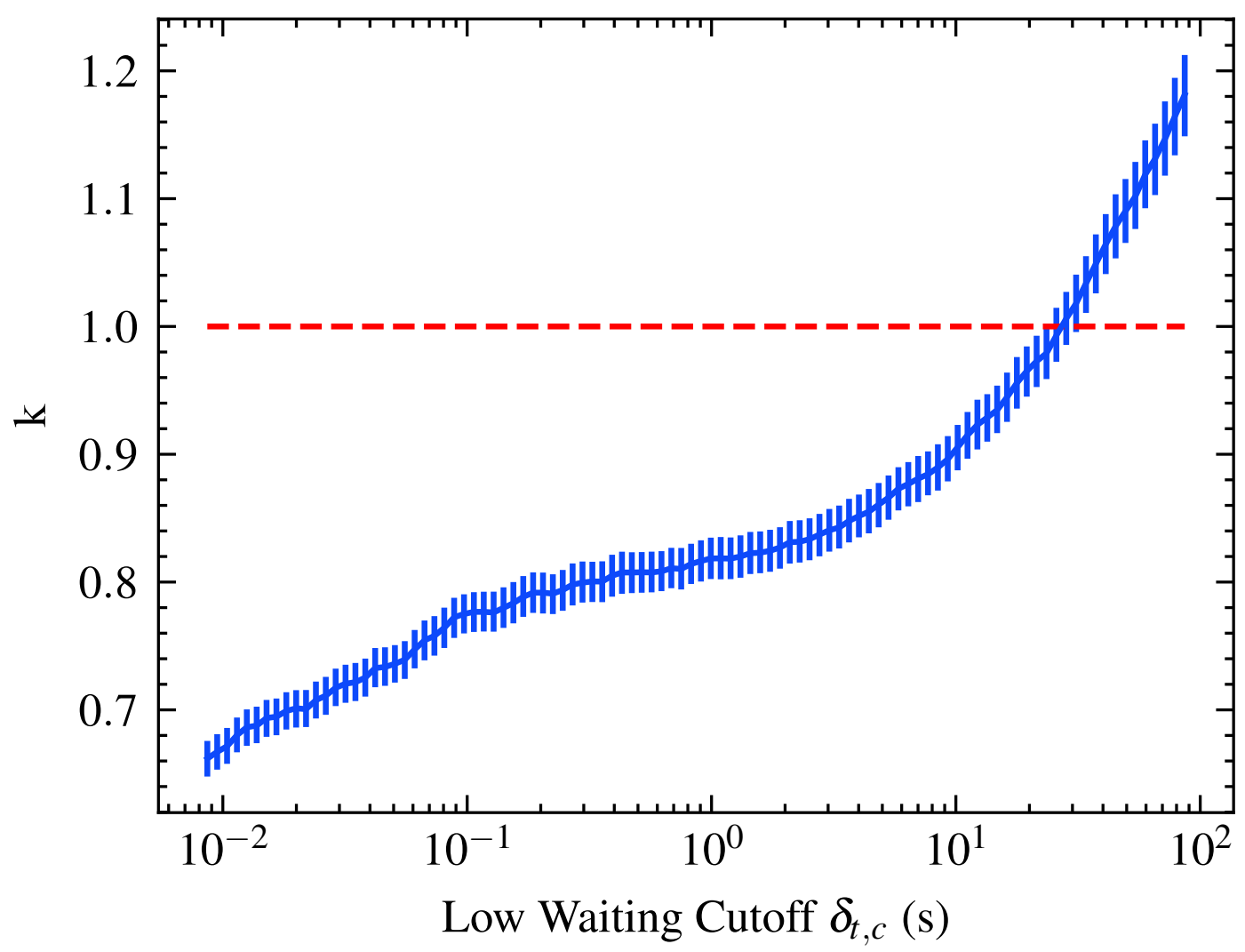

Figure 4. The shape parameter $k$ against the low-waiting-time cutoff $\delta_{t, c}$ for Weibull distribution. The best-fitting results of the Weibull function with $\delta_{t}>\delta_{t, c}$ are derived. We select some different $\delta_{t, c}$ and show the shape parameters $k$ in blue lines. The blue vertical lines are $1 \sigma$ uncertainties. The dashed red line is $k=1$. We found that the burst behavior approaches a Poisson process when $\delta_{t, c}=28 \mathrm{~s}$ (red-dashed line). 

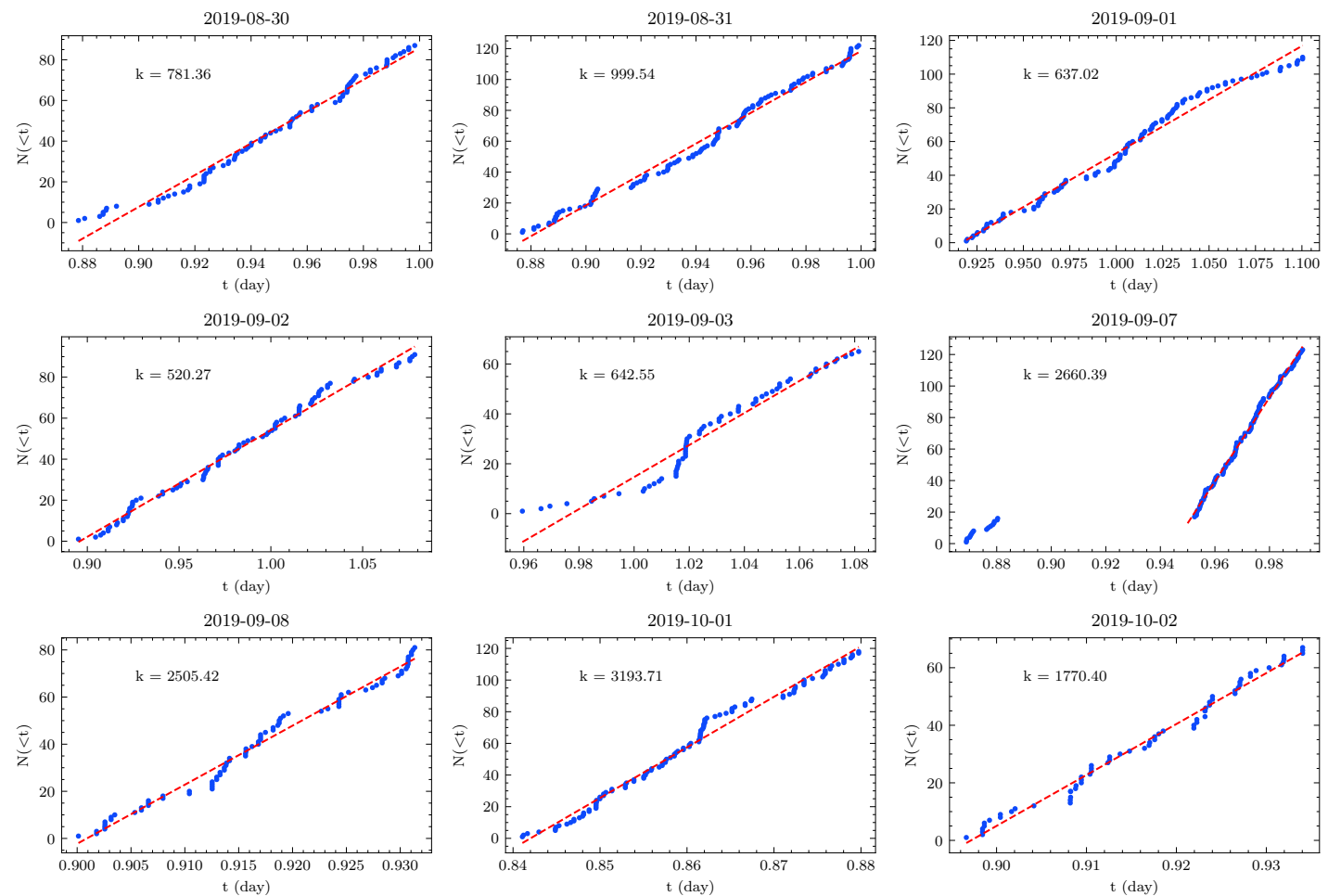

Figure 5. The cumulative distribution of burst number in single days. We select the days with the number of bursts greater than 60 . The x-axises are the decimal part of MJD burst time. In all cases, the distribution can be well fitted with $N(<t)=s t+b$, where $t$ is the burst time, and $s, b$ are fitting parameters. $N(<t)$ is the number of bursts with burst time earlier than $t$. The best-fitting results are shown as dashed red lines in each panel.
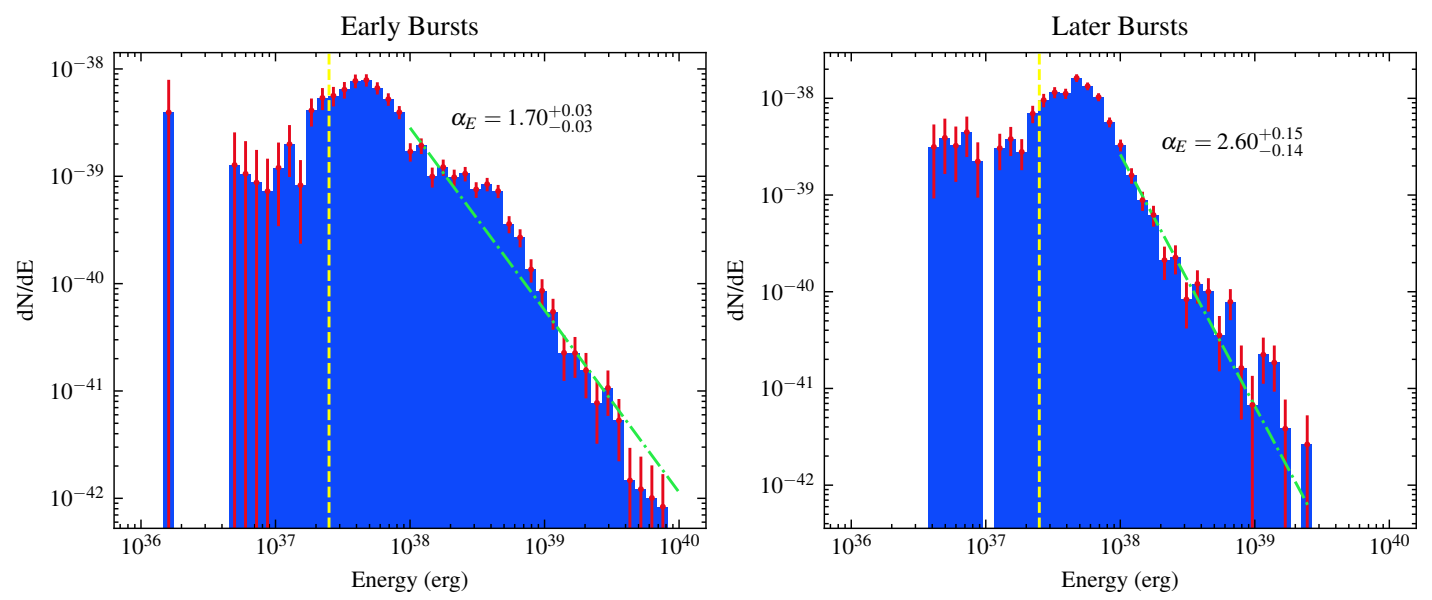

Figure 6. The energy distribution of bursts in different epochs. The early and late bursts correspond to the bursts occurring before and after MJD 58740, respectively. The vertical yellow dashed is the $90 \%$ threshold of FAST telescope. We use the simple power law to fit the bursts with energy greater than $10^{38}$ erg. The dot-dashed green lines are the best-fitting results. The power-law indices are also shown in each panel. 


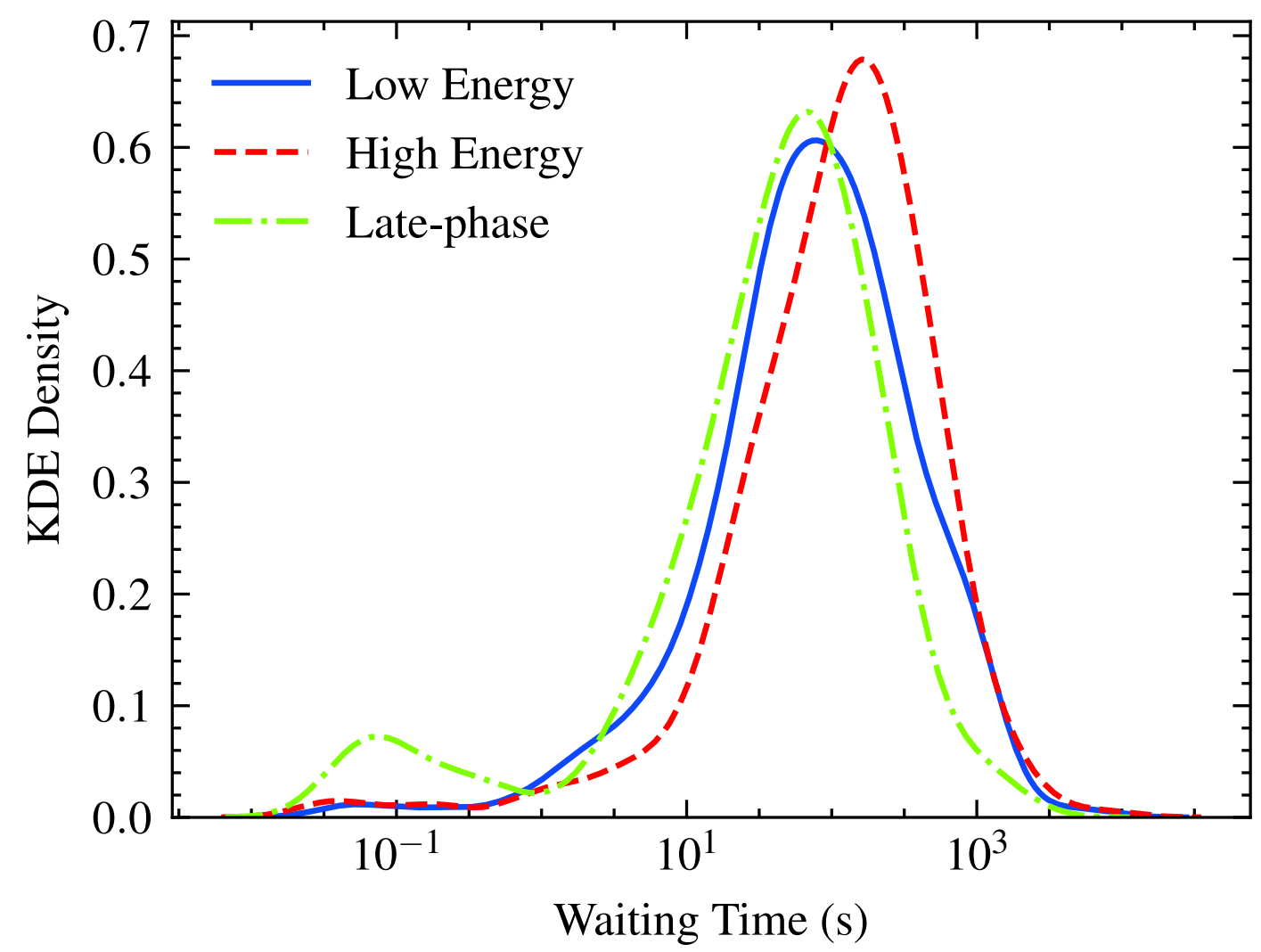

Figure 7. The KDE of waiting times for three sub-samples. According to the bimodal structure in Li et al. (2021a), we divide the bursts into 3 sub-samples: the low-energy bursts and high-energy bursts from MJDs 58717 to 58740 , and the late-phase bursts. The distributions of three sub-samples are similar, but with different median values, which are 78.19 s, $133.61 \mathrm{~s}$ and $54.62 \mathrm{~s}$ for low-energy bursts, high-energy bursts and late-phase bursts, respectively. 


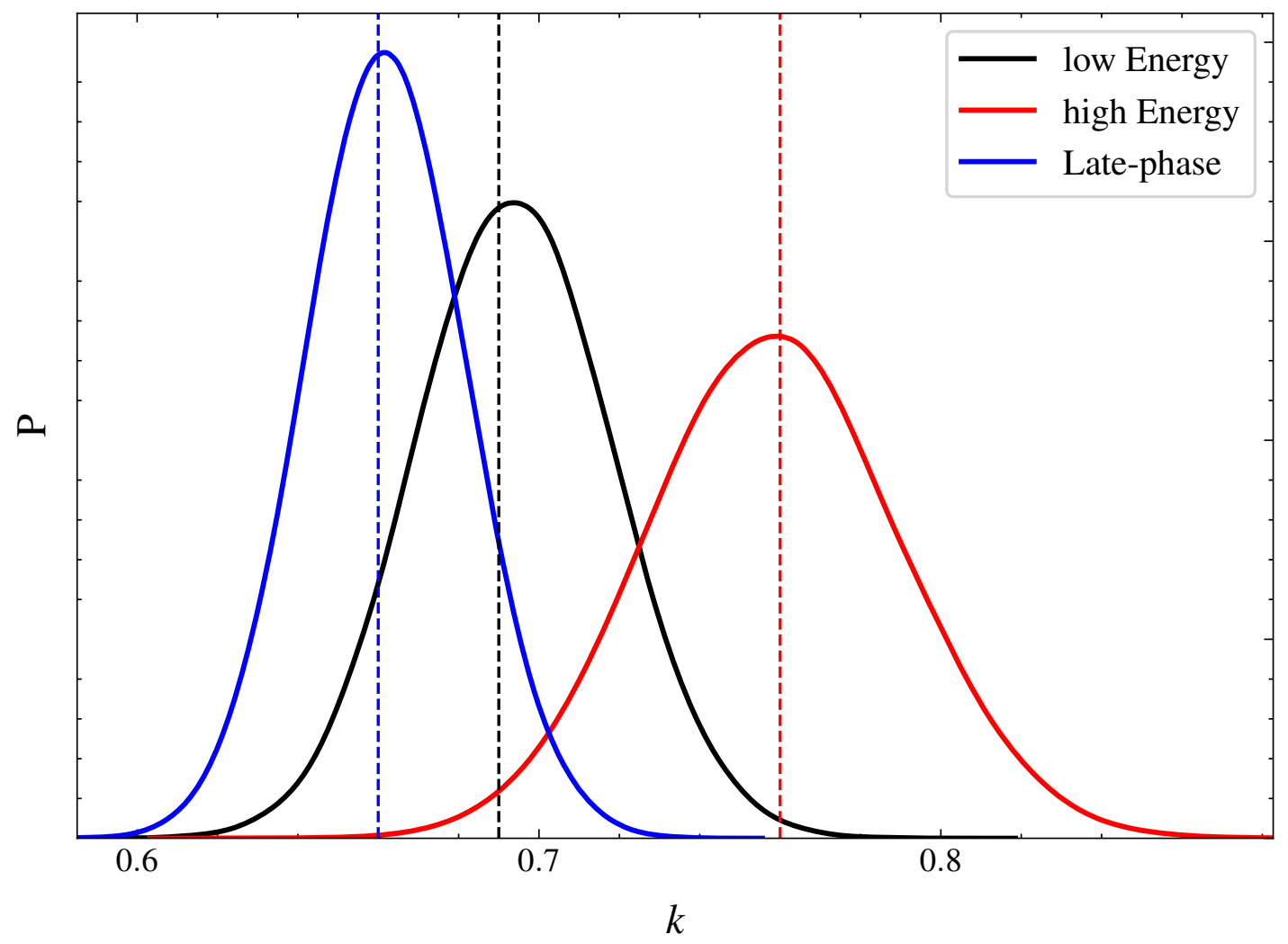

Figure 8. The shape parameter $k$ for the three sub-samples. The solid lines are the posterior probability distributions of the $k$ parameter for the three sub-samples, and the dashed lines indicate the mean values of the posterior distribution. The value of $k$ for low-energy bursts and late-phase bursts are consistent. However, the value of $k$ for high-energy bursts is slightly higher. 


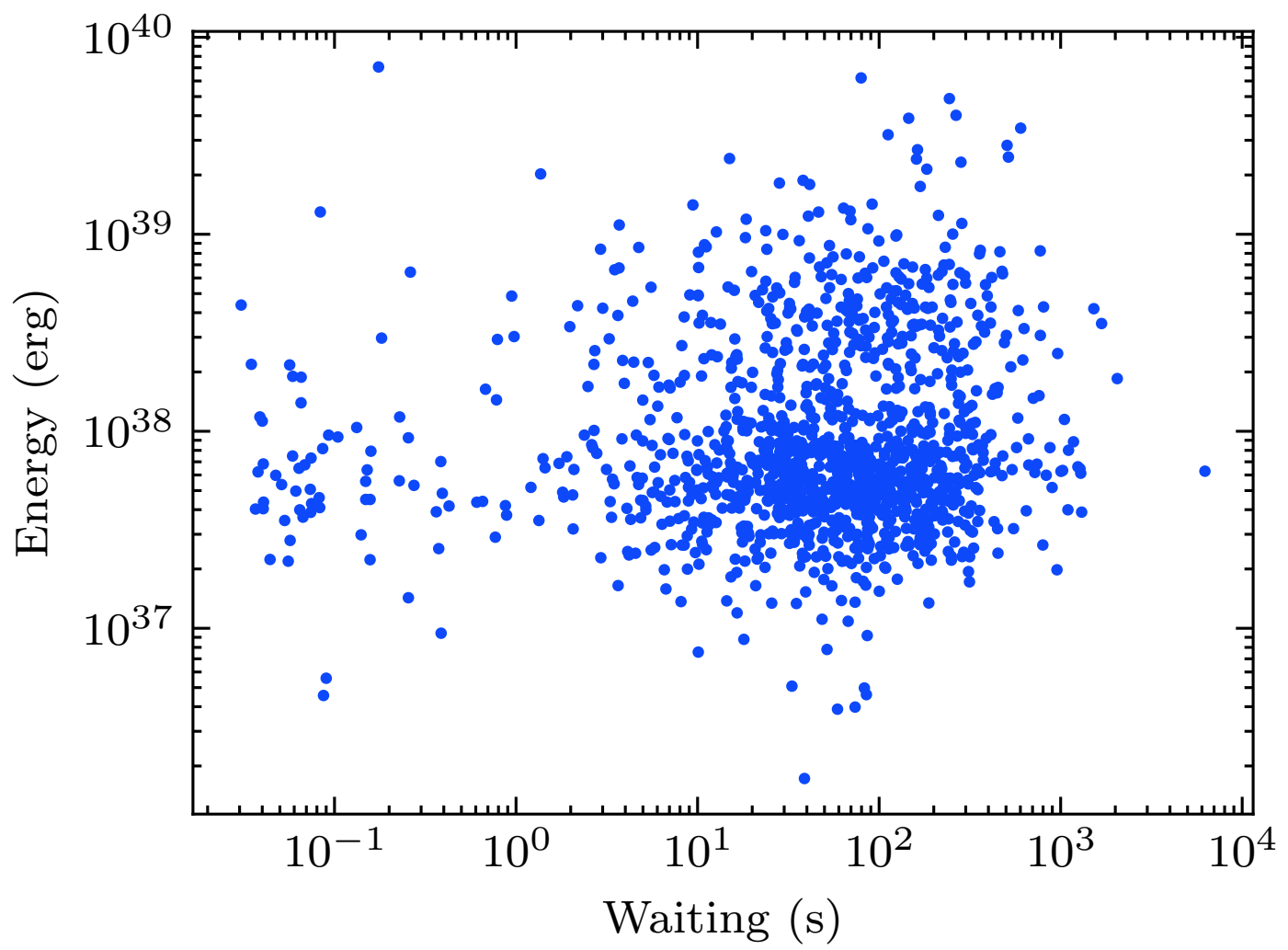

Figure 9. Scatter plot between waiting time and the energy of the burst after the waiting time. There is no correlation between burst energy and waiting time. 


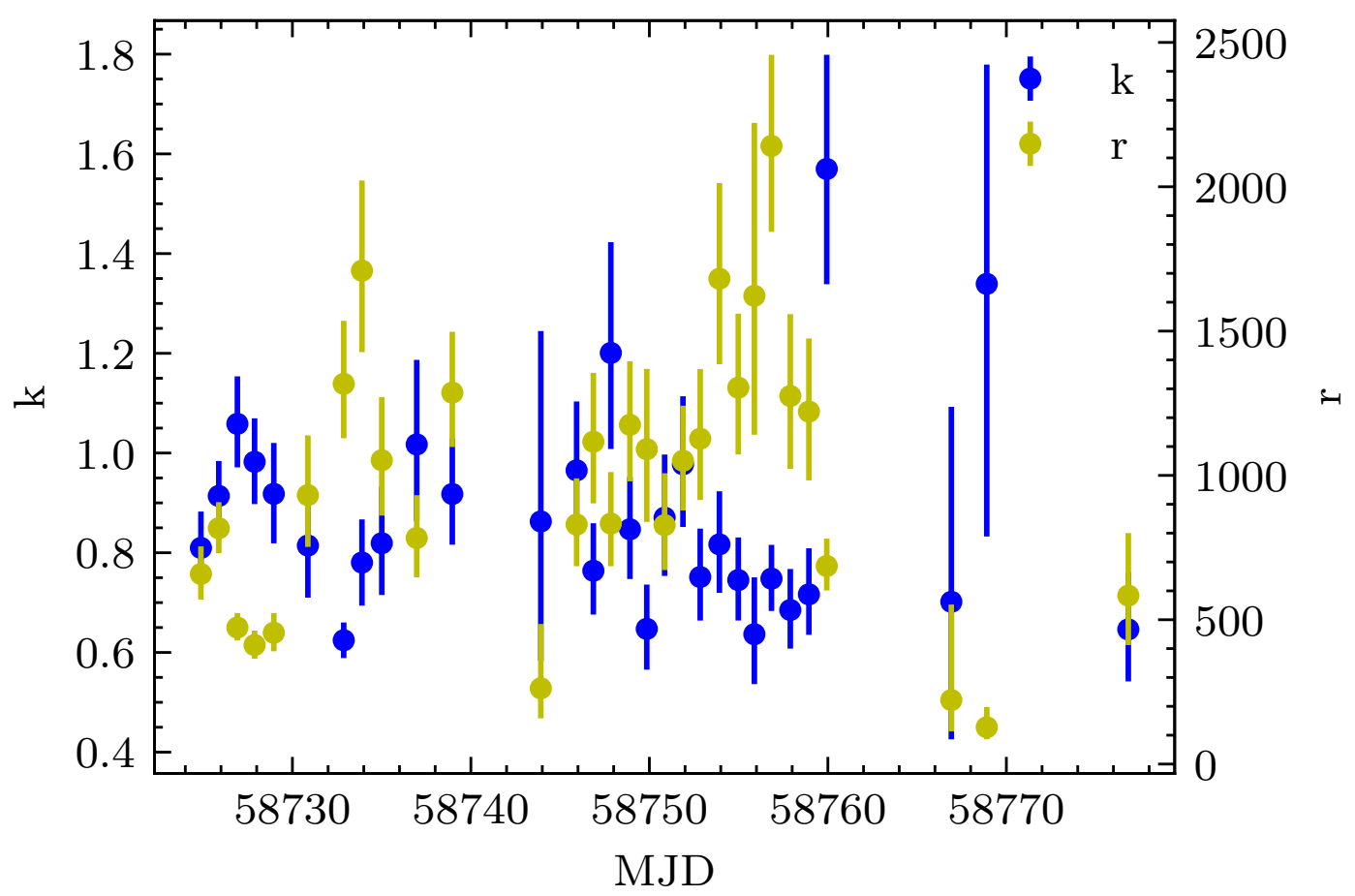

Figure 10. Shape parameters $k$ and burst rate $r$ in single days with the burst number greater than 5 . The blue points are the shape parameters $k$ with $1 \sigma$ errors, while the yellow points are the burst rate $r$ with $1 \sigma$ errors. 